\title{
Subjetividades, estructuras y procesos. Pensar las Ciencias Sociales Gastón Molina Domingo
}

\author{
Universidad Central de Chile, Santiago, Chile, 2012, \\ 355 págs.
}

\section{Raúl Rodríguez Freire}

Pontificia Universidad Católica de Valparaíso, Valparaíso, Chile.

Email: raul.rodriguez@ucv.cl

No hace mucho, estando presente en una reunión dedicada a la reestructuración de un programa de magíster en el que participo, se nos dijo que hoy en día un postgrado debe su existir a la idea de pertinencia. Esto es, cualquier programa, incluso uno de pregrado, debe tener como requisito el ser pertinente. ¿Cómo se define la pertinencia?, pregunté inmediatamente, pues la palabra no dice mucho... "a la matrícula”, fue la respuesta. Un eufemismo que no quiere referir directamente al mercado, pues es este el que finalmente termina decidiendo la pertinencia de una carrera universitaria o de un programa de postgrado, dado que será el número de alumnos inscritos lo que determinará su éxito o su fracaso. Pero si la RAE define por "pertinente" lo "Perteneciente o correspondiente a algo", en este caso, lo relativo al mercado, en su tercera acepción se nos señala que se trata de un adjetivo "Conducente o concerniente al pleito" y el pleito, me gustaría argumentar a partir de este libro que reseñamos, es lo relativo al pensamiento. Así ha sido por lo menos desde que Kant inscribiera la idea de conflicto en la política universitaria, al tiempo que abogaba por la libertad incondicional del pensar. Nuestro escenario supone entonces que la universidad contemporánea tiene, respecto de la hegemonía de las cifras, por impertinente al saber, cuestión que hace del pensamiento una verdadera anomalía.

Comienzo el presente comentario a partir de esta anécdota, pues ella contiene una línea a través de la que se podría leer Subjetividades, estructuras y procesos. Pensar las Ciencias Sociales, editado por Gastón Molina Domingo. Se trata de un libro que viene a inaugurar la colección Trazos, una publicación impulsada por la Facultad de Ciencias Sociales de la Universidad Central de Chile, con el cometido de problematizar conceptos clave inscritos en el corazón del pensamiento social. Así, en la solapa vemos que los libros que vienen se encargarán de abordar, uno, las nociones de evaluación, gestión y riesgo, triada neoliberal de relevancia en el actual gobierno del presente, mientras uno de los que continúa asumirá la memoria, relato y trauma, nociones centrales en el Chile postdictatorial.

En cuanto a Subjetividades, estructuras y procesos, consta de doce 
textos, estructurados en tres capítulos y articulados a partir de la triada conceptual que hace de título, introducidos por Molina, quien se encarga de hilvanar el conjunto acentuando la idea de pensar las ciencias sociales, donde el pensar es definido como anomalía: “cuando un enigma particular no entra en los cuadros conceptuales de la ciencia normal, cuando no puede ser clasificado y ordenado, surge la anomalía, es decir, que lo particular se transforma en singularidad allí donde se disloca toda clasificación posible” (p.15). Aquí el pensamiento vendría a dislocar, cuando no a suspender, la clásica (y metafísica) oposición que ha dominado gran parte del desarrollo de las ciencias sociales: sujeto v/s estructura y su variante lo general / lo particular. Pero, en tanto anomalía, el pensamiento hace algo más, pues su potencia no radica solo en deshacer los entuertos de la dualidad sino, y de manera fundamental, en desbaratar la lógica que funda la idea de conocimiento: "La inconmensurabilidad la encontramos en la tensión entre conocer y pensar. Conocemos cuando podemos poner lo particular bajo la regla general, pensamos cuando ya no contamos con la regla general y lo particular deviene en singularidad que se desvía de toda posible clasificación” (p.16). De manera que pensar las ciencias sociales es dejar de pensar en su gramática dominante, atreverse a obliterar el orden de los números y encarar la anomalía de la singularidad. Casi todos los ensayos reunidos en este libro comparten tal desafío, aunque no lo abordan de manera homogénea, dada la diversidad de disciplinas participando, más allá del campo circunscrito tradicionalmente por las ciencias sociales, pues la psicología, el trabajo social y la sociología son acompañadas por la historia, la filosofía y la crítica literaria.

Distribuidos de manera proporcional, cada capítulo consta de cuatro textos. Los primeros, podrían tener como común denominador el impacto del espaciamiento del tiempo, mientras el segundo es reunido en torno a la subjetividad, y el último pone en escena las tensiones que atraviesan a las ciencias sociales.

El primer capítulo, que lleva por título "Estructuras históricas y agencias individuales: la elaboración de la experiencia”, es el más extenso y reúne los trabajos de Pablo Aravena, Pablo Cabrera, Fernando Blanco y Danilo Martuccelli. Del primero me gustaría rescatar el tono que podemos ver compartido por quienes le siguen en el índice: "Hemos sido espectadores de como el impulso conmemorativo asociado al bicentenario ha retrotraído la historiografía a sus formulaciones más arcaicas y conservadoras: historia de grandes hombres, historia de batallas, historia nacional”, sentencia Aravena, y se pregunta, en consecuencia, por los rastros de la “operación histórica” (p.29), esto es, por la operación del pensar, ausente completamente en tiempos conmemorativos, tiempos donde el pasado ya no es el régimen de historicidad dominante, sino el presente, razón por la cual urge preguntarse, con François Hartog, por la historia que se podría hoy hacer (p.30). Desde el psicoanálisis, a igual punto, la hegemonía del presente, llega Cabrera, aunque agrega, a modo de recordatorio, que en este presente "el dolor y la muerte, la contradicción y la crisis son lo común a nuestras sociedades, e inherentes a los logros y conquistas del capitalis- 
mo” (p.76), modo de producción que, en su versión neoliberal, da lugar además a una sociedad narcisista (p.77). Preocupado por los cambios públicos y privados acaecidos en el Chile postdictatorial, por esta misma senda avanza el ensayo de Blanco, pues su preocupación también pasa por "la disolución de las narrativas históricas” (p.109), y su reemplazo por una lógica de la actualidad que colma el mercado cultural de instantaneidad (p.143). Como tales narrativas se estructuraron a partir de conceptos que han perdido su fuerza heurística, hoy el aparato categorial de las Ciencias Sociales se nos presenta completamente arruinado (p.114). Pero, como también señala Cabrera, la perdida de los tiempos pretéritos y futuros también implica la interrupción de la experiencia subjetiva a que dio lugar la modernidad: "La caída de la memoria, como sostén simbólico privilegiado de la experiencia histórica, impide el anclaje de estos sujetos en lo social” (p.118), afirma Blanco. En cuanto al artículo de Martuccelli, lo suyo es más bien un mapa o una cartografía de la sociología contemporánea, cuya heterogeneidad conceptual bien podría intersectarse en la idea de contingencia, pues a ella intentan responder "la mayor parte de las variantes actuales de teoría sociológica": "Es la contingencia que, se use o no el término, es lo que esta' en el corazón del nuevo pathos especifico de la teoría social, y que hace que el mosaico sociológico contemporáneo presente una diferencia mayor en relación a un pasado aún cercano” (p.173).

En segundo capítulo, "Lugares de la subjetividad individual”, reúne trabajos de Kathya Araujo, José Fernando García, Sergio Rojas y JeanLouis De`otte, y se podría señalar que desde el lugar del individuo, la ficción de la naturalización es lo que se presenta como objeto de cuestionamiento compartido, aunque también se pone el acento en la necesidad de renovar las herramientas teóricas y metodológicas (p.187), a la vez que se acentúa la complejidad del presente. Araujo, en lo que es una de sus reflexiones más interesantes, da cuenta de la relevancia del feminismo para la comprensión del individuo, dado que este "se constituye en su meta normativa en vinculación estrecha con el objetivo político de reconocimiento e incorporación de las mujeres a la polis” (p.191), con lo cual quiere referirse un activo trabajo de individuación por parte de los mismos sujetos. En cuanto a García, pone en cuestión el lugar que Habermas le entrega a la lógica, en tanto razón y método, para terminar afirmando "la función fundamental que juega la claridad hermenéutica", dado que esta "es condición inescapable de la razón”, algo que el sociólogo alemán tiende a desconsiderar.

Sergio Rojas, aportando con uno de los textos más importantes del libro, determina una idea de malestar producida por el mismo orden imperante. En otras palabras, el malestar con el neoliberalismo es, también, un malestar producido por el neoliberalismo mismo. Las implicancias de esta reflexión deben ser trabajadas detenidamente, pues de estar en lo cierto, y creo que lo está, se deben volver a pensar las estrategias políticas que están realizando los movimientos sociales del siglo XXI.

Articulando representación (fotografías) y política con la cuestión de la individuación, Jean-Louis Déotte da cuenta de la importancia de la 
muerte para las relaciones entre los vivos, un proceso que la desaparición forzada interrumpiría de manera traumática. De ahí que las fotografías, en este caso de Omar Daoud, fotógrafo argelino, tenga como objetivo "restaurar la posibilidad de un intercambio simbólico entre los vivos y los muertos, y no de edificar monumentos a los muertos” (p.266).

En cuanto al tercer capítulo, “Nuevos espacios sociales”, integrado por Carlos Montan o, María Emilia Tijoux, Jorge Mario Flores / Georg Unger Vergara, Cecilia Aguayo Cuevas, encontramos en él trabajos que abordan los dilemas y tensiones de las ciencias sociales en su vínculo con lo que hoy, gracias a Michel Foucault, llamamos gubernamentalidad. Ello porque la psicología comunitaria y el trabajo social operan en gran medida reproduciendo el sistema neoliberal. Ello conlleva la necesidad, en palabras de Montaño, de “construir colectivamente un "proyecto ético-político profesional, que pueda enfrentar con competencia y compromiso... las condiciones en que viven los trabajadores (con y sin empleo) y demás sectores subalternos" (p.271). Para ello, señala Tijoux, "descubrir lo que se oculta tras [las] miserias que continuamente se exponen como 'dadas"” (p.306), se vuelve un imperativo político de primer orden. Se trata este de un punto que nos conecta con los primeros textos, al insistir en que las ciencias sociales críticas, anómalas, deben trabajar por mostrar que la naturalización del orden neoliberal es una ficción que puede y tiene que ser deshecha, aun más cuando nociones como la de comunidad no se opone al individualismo constituido por la acumulación del capital, sino, como el malestar, refiere un lenguaje técnico del gobierno del presente, tal como muestran Flores y Unger (p.326). Así, las tensiones entre lo epistémico y lo ético, al decir de Aguayo (p.345), el saber que de ellas se encarga, están todavía en deuda por parte del trabajo académico.

En conjunto, los doce trabajos reunidos en Subjetividades, estructuras y procesos. Pensar las Ciencias Sociales, dan cuenta del estado actual de las disciplinas reunidas bajo este campo, lo que hace del libro que comentamos una excelente síntesis de los desafíos que afrontamos quienes nos dedicamos al pensamiento anómalo en medio de una universidad centrada en la administración del saber y su disciplinamiento desde los números. 